\title{
Treatment planning for multicatheter interstitial brachytherapy of breast cancer - from Paris system to anatomy-based inverse planning
}

\author{
Tibor Major, MSc, PhD, Prof. Csaba Polgár, MD, PhD \\ Center of Radiotherapy, National Institute of Oncology, Budapest, Hungary
}

\begin{abstract}
In the last decades, treatment planning for multicatheter interstitial breast brachytherapy has evolved considerably from fluoroscopy-based 2D to anatomy-based 3D planning. To plan the right positions of the catheters, ultrasound or computed tomography (CT) imaging can be used, but the treatment plan is always based on postimplant CT images. With CT imaging, the 3D target volume can be defined more precisely and delineation of the organs at risk volumes is also possible. Consequently, parameters calculated from dose-volume histogram can be used for quantitative plan evaluation. The catheter reconstruction is also easier and faster on CT images compared to X-ray films. In high dose rate brachytherapy, using a stepping source, a number of forward dose optimization methods (manual, geometrical, on dose points, graphical) are available to shape the dose distribution to the target volume, and these influence dose homogeneities to different extent. Currently, inverse optimization algorithms offer new possibilities to improve dose distributions further considering the requirements for dose coverage, dose homogeneity, and dose to organs at risk simultaneously and automatically. In this article, the evolvement of treatment planning for interstitial breast implants is reviewed, different forward optimization methods are discussed, and dose-volume parameters used for quantitative plan evaluation are described. Finally, some questions of the inverse optimization method are investigated and initial experiences of the authors are presented.

J Contemp Brachytherapy 2017; 9, 1: 89-98 DOl: https://doi.org/10.5114/jcb.2017.66111
\end{abstract}

Key words: APBI, breast cancer, interstitial, multicatheter, treatment planning, dosimetry.

\section{Purpose}

Brachytherapy as a boost or monotherapy plays an important role in treating breast cancer [1,2]. In the recent years, accelerated partial breast irradiation (APBI) with its 4-5 day treatment course has been widely used worldwide to treat early stage breast cancer patients. Different techniques are available for APBI with brachytherapy (BT) and external beam radiation therapy (EBRT) [3]. Brachytherapy techniques include interstitial implants, intracavitary devices with single/multilumen balloons, hybrid applicators, and irradiation with low energy X-ray $[1,2]$. External beam radiation therapy can be performed with 3D conformal radiotherapy (3D-CRT), intensity modulated radiation therapy (IMRT), volume modulated arc therapy (VMAT), proton therapy, and intraoperative electron irradiation [4]. Among all APBI techniques, currently multicatheter interstitial brachytherapy (MIBT) has the longest patient follow-up and has a Level 1 evidence as a valid treatment alternative to whole breast irradiation after breast conserving surgery [5]. Recommendations for APBI patient selection and treatment workflow were published in Europe and in the USA $[6,7,8]$. Recently, the GEC-ESTRO (Groupe Européen de Curiethérapie European Society for Radiotherapy and Oncology) Breast Cancer Working Group has compiled guidelines for target definition and delineation for partial breast irradiation using MIBT after breast conserving closed and open cavity surgery $[9,10]$.

In classical breast brachytherapy, treatment planning was based on two X-ray films. Using different reconstruction methods (e.g., orthogonal, variable angle, semi-orthogonal) and a digitizer device, catheters were reconstructed in three dimensions (3D), but the anatomical information about the patients was very limited. The surgical clips approximately showed the position of the lumpectomy cavity, and the catheter fixation buttons indicated the skin surface in a number of points only. At that time, neither dose coverage nor conformality was possible to be determined, and it was assumed that the catheters are in the right positions. Later, with the introduction of cross-sectional imaging, the real 3D dose planning became available. The reconstructed catheters geometry and the delineation of target volume and organs
Address for correspondence: Tibor Major, PhD, Center of Radiotherapy, National Institute of Oncology, Budapest, 7-9 Ráth György u., Budapest, Hungary, H-1122, phone: +36 1 224-8600, fax: +36 1 224-8620,

๑ e-mail: major@oncol.hu
Received: 05.01.2017

Accepted: 10.02.2017

Published: 28.02.2017 
at risk on computed tomography (CT) slices made possible for conformal irradiation. But, at the beginnings, the post-implant CT imaging was used only for evaluating dose plans, and just a few quality indices were calculated $[11,12,13,14]$. These studies clearly demonstrated that the conventional fluoroscopy based implantations usually could not result in acceptable dose distributions regarding all dose parameters. Cuttino et al. [15] reported significantly increased target coverage and dose homogeneity after implementing their CT-guided implantation technique. In a paper by Major et al. [16], 91\% target coverage and 0.33 for dose non-uniformity ratio was published evaluating 28 CT-based treatment plans. These values were significantly better than the corresponding data of the radiography-based implantation method. Nowadays, based on the persuading early dosimetric results, CT-based treatment planning has become the gold standard for interstitial BT of breast cancer.

The aim of this article is to review treatment planning issues of multicatheter interstitial brachytherapy and share our experience in forward and inverse planning methods.

\section{Material and methods}

\section{Pre-planning imaging}

Since even the most advanced optimization technique cannot compensate for a wrong implant geometry, it is of the utmost importance to place the catheters in the right positions. In order to get an acceptable dose distribution, the planning target volume (PTV) has to be geometrically covered by the catheters. To achieve this, different imaging methods can be used. With ultrasound (US) guidance, the deepest needles can be inserted manually if the resection cavity is visible, and then the rest of catheters are placed with a template, which can ensure an even spacing between catheters. Another approach is when the superficial needles are inserted as first under US guidance followed by the deeper needles with template guidance, but the artefacts from the shallow needles may cause vis-

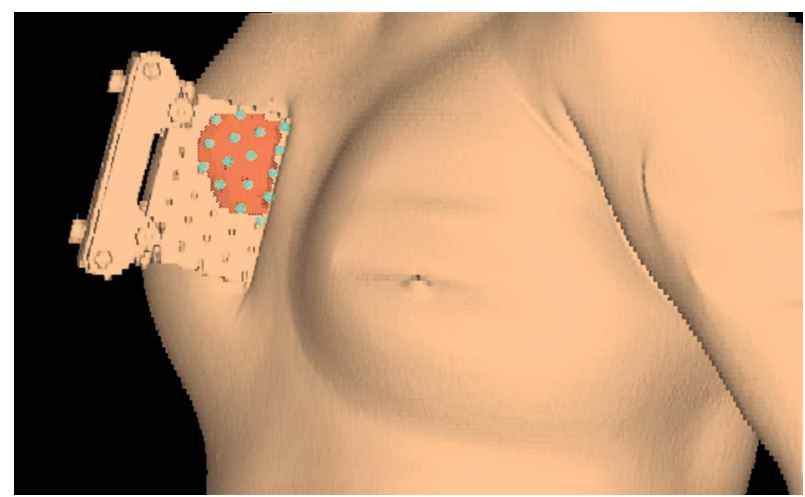

Fig. 1. 3D rendering of patient anatomy with a template on the right breast in needle's-eye-view after pre-implant computed tomography imaging. The red colored planning target volume is projected into the template with the holes and the light blue dots show the planned positions of the catheters ibility problem [17]. If the needles are manually inserted using US imaging, the PTV contour and all entry and exit points have to be marked on the skin surface in order to plan the needles distribution. However, the best method is when a $3 \mathrm{D}$ imaging with full anatomical information about the resection cavity, PTV, and organs at risk (OARs) is available before the insertions. Then, the number and positions of the catheters can be determined using a 3D rendered image with a template placed around the breast (Figure 1). If the intention of the treatment is delivering a boost dose, steel needles can be used with 1-3 fractions, but in monotherapy treatments with 7-10 fractions, the needles are replaced with plastic catheters for the sake of patient's convenience.

\section{Planning procedure}

However, any imaging technique is used before the implantation, after the insertions of the catheters, a new CT imaging is needed for planning purpose. Generally, the patient is positioned on the CT table in the same way as for EBRT making sure the same anatomical conditions at boost treatment as for external irradiation. Generally, $3 \mathrm{~mm}$ slice thickness is acceptable for accurate catheter reconstruction and target delineation. Organs at risk and resection cavity must be outlined, and the PTV should be created according to the available guidelines [10]. Using special markers in the catheters with good visibility but without artefact is a great help during catheter reconstruction (Figure 2). Nevertheless, without markers, the catheters can be also visualized merely by the inside air (Figure 2), and with proper windowing, the reconstruction can be properly performed. In this case, it is important to see and identify the fixation button at the distal end of each catheter, since the first possible dwell position has to be related to it. The catheter reconstruction can be performed in axial, sagittal, coronal planes, or even in oblique planes, which have been created parallel with or orthogonal to the catheters (extra coordinate system - ECS). Catheters should be numbered or uniquely labelled, so that each

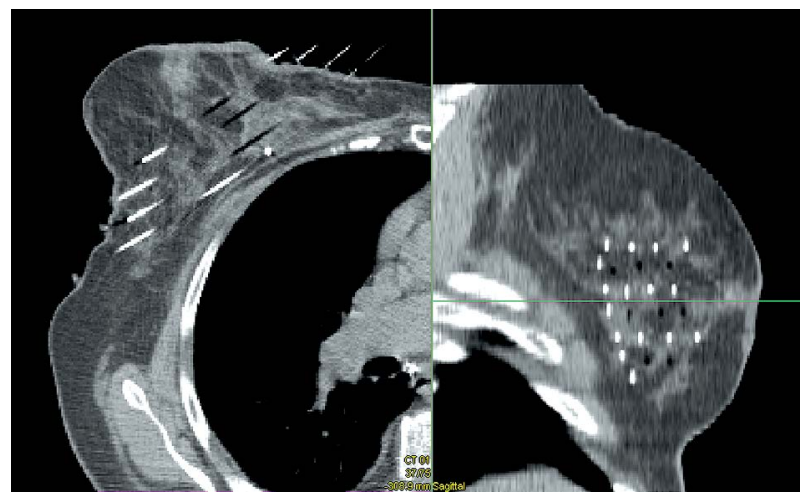

Fig. 2. A transversal and sagittal computed tomography slice with implanted catheters. In most of the catheters, special markers are inserted (shown with white lines/ dots), but some of them do not have any markers and only the inside air makes them visible (shown with black lines/ dots) 
individual catheter can be identified. The active source positions inside the PTV can be defined manually or automatically with or without margin inside or outside the target surface, if needed. In classical BT planning using the Paris dosimetry system (PDS), the basal dose points in the central plane play an important role. The central plane can be easily created using the ECS in the CT-based planning. The midpoints between catheters (basal dose points) can be placed manually by visual inspection, or automatically by a software if the catheters geometry is regular. After normalizing the dose distribution $(100 \%)$ to the mean central dose (MCD in Paris system), which is the mean dose in basal dose points, the isodose level for prescription can be selected using a special multiplication factor between 0.1 and 10. For example, using the factor of 0.85 , the dose will be prescribed to the $85 \%$ isodose level corresponding to the PDS. To calculate the treatment time, the absolute dose and the fraction number must be specified. Theoretically, the PDS provides homogeneous dose distribution, but the target coverage and conformality may not be sufficient enough. To increase the coverage lower than $85 \%$, isodose level can be selected for dose prescription, but the worsening homogeneity must be taken into consideration. In the contrary, if increasing the isodose level for prescription, the dose distributions will be more homogeneous, and the target coverage decreases simultaneously. Another way to manage the target volume coverage and homogeneity of dose distribution is applying several dwell time optimization methods.

\section{Dwell time optimization methods}

Various dose optimizations are available in BT using a stepping source (Table 1). The classical Paris dosimetry system can be imitated by using uniform dwell times in all dwell positions, which means no optimization is applied. In that case, the dose distribution is always homogeneous, but the conformality will not be optimal [18]. The target volume can be covered by the prescription isodose surface adequately, but relatively large volume of normal tissue will be unnecessarily irradiated by the prescription dose (PD). This is the consequence of the extending active lengths in the catheters out of the target volume, which is required in the PDS. For this reason, usually the classical PDS is not used in breast BT. However, the concepts of its implant geometry and dose prescription are still employed even if the dwell times are calculated with a forward optimization method. In most optimization methods, the 3D target volume is not used directly during optimization; only in plan evaluation, the DVH parameters are related to the PTV.

Changing the individual dwell times or weight factors manually in a spreadsheet is not an effective method to shape the dose distribution. It is rather time consuming and recommended only for small local adjustments. After geometrical optimization (GO), the resulting dose distribution will always be homogeneous. Another advantage is that there is no need to define dose points for optimization because the source dwell positions themselves will serve as reference dose points. The dwell time at any dwell position will be inversely proportional to the dose delivered by the other dwell positions. The consequence is the increased dwell times at the ends of the catheters, and in dwell positions in deviating catheters [19]. If the catheters geometrically cover the target volume properly, the reference isodose surface will always include the PTV. Since the dose distribution is related only to the catheters, if there is a geometric miss, a significant underdosed region may develop in the PTV. By selecting a lower isodose level for dose prescription, the target coverage can be slightly improved, but the dose to normal tissues may increase. At polynomial optimization, an objective function is minimized, which describes the difference between the calculated and required dose values in specified dose points. These points can be placed at a distance outwards of or in mid-positions between the catheters (distance or volume dose points). Using distance dose points, it is assumed that they are placed on the surface of the target volume. With volume dose points, we can optimize the doses in central points between the catheters in the whole volume and not only in the central plane. This method is called Stepping Source Dosimetry System (SSDS) and is the extension of the PDS [20]. Regarding dosimetry, due to the stepping source, the SSDS is superior to the PDS [21]. In the SSDS, the dose can be prescribed for a proper isodose level similarly to the GO. When the PTV is defined three-dimensionally, we can place dose points on its surface and can optimize and prescribe the dose $(100 \%)$ on them (conformal dosimetry system). With this method, even if the target volume is irregular, very conformal dose distribution can be obtained if the catheters are evenly distributed inside the volume. Since the dose homogeneity is not taken into account during optimization, the dose homogeneity will be unacceptable, since large high dose volumes develop inside the PTV [16]. The graphical optimization (GRO) is an attractive way to change the shape of an isodose curve locally

Table 1. Optimization methods in high-dose-rate brachytherapy

No optimization (Paris dosimetry system - PDS)

Uniform dwell times

Forward optimization

Manual optimization (editing dwell times/weights)

Geometrical optimization (on distance and volume)

Polynomial optimization

Distance dose points (dwell time gradient restrictions)

Volume dose points (SSDS ${ }^{1}$ - extension of PDS)

Dose points on the target surface (conformal dosimetry system)

Graphical optimization (only for local adjustments)

Inverse optimization (IPSA ${ }^{2}, \mathrm{HIPO}^{3}$ )

Anatomy based with surface and volume dose objectives

${ }^{1}$ Stepping source dosimetry system, ${ }^{2}$ Inverse planning simulated annealing, ${ }^{3}$ Hybrid inverse planning optimization 
or globally. On the screen with the cursor, we can select an isodose line, and with the "drag and drop" method, the isodose line can be shifted into the desired position. If the local adjustment option is selected, only the nearby dose distribution will be changed, while with the global option, the dose distribution will change isotropically in $3 \mathrm{D}$. The coverage and conformality can be easily improved with GRO, but it must not be forgotten that at the same time, the homogeneity will deteriorate. In many cases, the GO followed by GRO results in acceptable dose distribution, since the GO can provide the homogeneity, and with the GRO, the coverage can be further increased.

Nowadays, inverse optimization is already available in BT treatment planning. The key point in inverse optimization is that all dosimetric requirements (dose coverage, dose homogeneity, and organs at risk protection) are simultaneously and automatically taken into account in the planning process. In the first step, the clinical objectives are translated into minimum or maximum dose limits and importance factors, and then, the source dwell times are calculated with minimizing objective functions, describing the differences between the calculated and required doses. The prerequisites for inverse optimization are as follows:

- reconstructed catheters geometry,

- delineated PTV and OARs,

- defined active source dwell positions,

- dose objectives for PTV, OAR-s and normal tissue.

Different optimization methods consider different mathematical descriptions for optimal plan.

The two most common inverse algorithms are the HIPO (Hybrid Inverse Planning Optimization) and IPSA (Inverse Planning Simulated Annealing) [22,23,24]. The former uses deterministic, while the latter works with stochastic method. HIPO is implemented in the Oncentra Brachy version 4.3 planning system (Nucletron, an Elekta company, Elekta AB, Stockholm, Sweden), where the target coverage and dose homogeneity are controlled by minimum and maximum dose values in the PTV, respectively. In a clinical case, Figure 3 shows an example for the input parameters for the HIPO. The minimum value in the PTV is $100 \%$ corresponding to the PD, while the maximum value is selected $150 \%$ of the PD. During optimization, the volume receiving maximum $150 \%$ of the PD is minimized. Furthermore, with a weight factor, the importance of the given requirement can be taken into account. Regarding protection of normal tissue and
OARs, maximum doses can be specified for them, which are also taken into consideration during optimization.

\section{Plan evaluation}

In classical BT, the plan evaluation was limited, having dose values in reference points and looking at the 2D dose distributions related to catheters/applicators. The use of these evaluation methods is still recommended, but additional tools are now available to assess the quality of the implant. Visual inspection of dose distribution on CT slices including sagittal and coronal reconstructions is still mandatory, however, the large number of CT slices and outlined organs make the evaluation quite subjective. For an objective assessment, quantitative parameters have to be employed. Table 2 shows the most common dose-volume parameters used in interstitial BT. Implant related parameters can always be calculated, even if the target volume is not defined. They characterize the size of the irradiated volume and homogeneity of the dose distribution. In optimal implant, the DNR is minimal and the DHI is maximal, since they are complementary indices, namely DHI $=1-$ DNR. When 3D PTV is defined, additional parameters can be calculated. The percentage of PTV receiving $\mathrm{xx} \%$ of the PD is denoted by Vxx. Additionally, the DHI can be determined for the target, when volumes in the PTV only are taken into account. In the relative homogeneity index (HI), volume receiving a dose between $100 \%$ and $150 \%$ of the PD is related to the volume of the PTV. Although only small differences occur between the various DHIs, its exact definition always must be given when used for reporting dose homogeneity and for intercomparisons of different plans. The overdose volume index (OI) characterizes the dose homogeneity with volume irradiated by $2 \times$ PD, related to volume of PTV. In an ideal implant, the DHI is large and the OI is small. The coverage index $(\mathrm{CI})$ is the fraction of the PTV receiving at least the PD. The conformal index (COIN) considers the coverage of the PTV by the PD (PTV $\left.\mathrm{PD}_{\mathrm{PTV}} / \mathrm{V}_{\mathrm{PTV}}\right)$, and also the unwanted irradiation of normal tissues outside the PTV $\left(\mathrm{PTV}_{\mathrm{PD}} /\right.$ $\mathrm{V}_{\mathrm{PD}}$ ) [25]. $\mathrm{PTV}_{\mathrm{PD}}$ is the volume inside the PTV irradiated by the $P D$, and the $\mathrm{V}_{\mathrm{PD}}$ is the volume receiving at least the PD. The definition of COIN can be extended to critical structures also, but this formalism is rarely used in breast $\mathrm{BT}$, since the OARs nearly always receive less dose than the PD. The dose distribution is most conformal when the COIN is maximal and is as close to 1 as possible. The external volume index (EI) is also related to conformality;

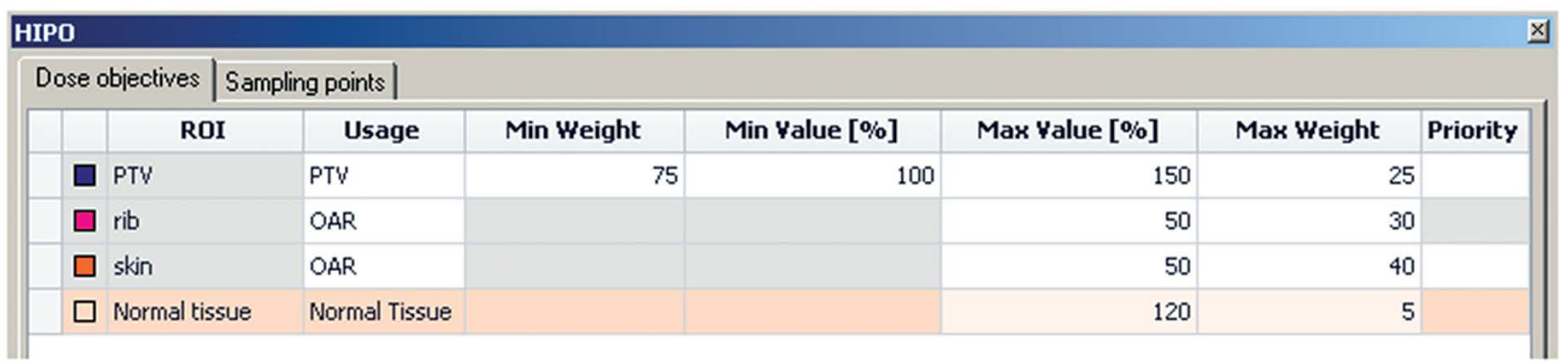

Fig. 3. Input parameters for optimization in HIPO. The Min Value and Max Value refer to relative doses, while the Min Weight and Max Weight mean relative importance factors 
Table 2. The most common dose-volume parameters used in interstitial brachytherapy

\begin{tabular}{|c|c|}
\hline Parameter & Definition/calculation \\
\hline \multicolumn{2}{|l|}{ Implant related } \\
\hline$V_{\text {ref }}$ & Absolute volume irradiated by reference dose \\
\hline$V_{1.5 \times r e f}$ & Absolute volume irradiated by $1.5 \times$ reference dose \\
\hline DNR (dose non-uniformity ratio) & $V_{1.5 \times \text { ref }} / V_{\text {ref }}$ \\
\hline DHI (dose homogeneity index) & $\left(\mathrm{V}_{\text {ref }}-\mathrm{V}_{1.5 \times \mathrm{ref}}\right) / \mathrm{V}_{\text {ref }}$ \\
\hline \multicolumn{2}{|l|}{ Target related } \\
\hline $\mathrm{V}_{\text {PTV }}$ & Volume of the PTV \\
\hline $\mathrm{Vxx}$ & Percentage of PTV receiving $x \mathrm{x} \%$ of the $\mathrm{PD}$ \\
\hline DHI (dose homogeneity index) & $\left(V_{100}-V_{150}\right) / V_{150}$ \\
\hline HI (relative homogeneity index) & $\left(V_{100}-V_{150}\right) / 100$ \\
\hline OI (overdose volume index) & $V_{2 \times P D} / V_{\text {PTV }}$ \\
\hline Cl (coverage index) & $V_{100} / 100$ \\
\hline COIN (conformal index) & $P T V_{P D} / V_{P T V} \times P T V_{P D} / V_{P D}$ \\
\hline El (external volume index) & $\left(\mathrm{V}_{\mathrm{PD}} / \mathrm{V}_{\mathrm{PTV}}\right)-\mathrm{Cl}$ \\
\hline Dxx & Percentage dose that covers $x \mathrm{x} \%$ of the PTV \\
\hline \multicolumn{2}{|l|}{ OAR related } \\
\hline $\mathrm{D}_{\text {mean }}$ & Mean dose in organ \\
\hline $\mathrm{V}_{\mathrm{xGy}}$ & Relative volume receiving $x$ Gy \\
\hline $\mathrm{D}_{\mathrm{xcm}}{ }^{3}$ & Relative dose given to most exposed $x \mathrm{~cm}^{3}$ of organ \\
\hline
\end{tabular}

$P D$ - prescribed dose, $P T V_{P D}$ - volume in PTV received at least the $P D$

it is the ratio of the normal tissue volume outside the PTV receiving at least the PD, to the volume of the PTV. Dose that irradiates certain part of the PTV is also used, e.g. D10 means the relative dose that irradiates $10 \%$ of the PTV.

Depending on the type of the OAR, the mean dose, volume irradiated by a given relative (V5), absolute dose $\left(\mathrm{V}_{5 \mathrm{~Gy}}\right)$, or dose irradiating a small volume (eg. $\mathrm{D}_{0.1 \mathrm{~cm}^{3}}$, $D_{2 \mathrm{~cm}^{3}}$ ) are generally reported. In several studies, the maximum point dose is calculated on the skin surface or in other organs. However, recently in CT-based planning, volumetric doses are already recommended to be used, e.g. $\mathrm{D}_{0.01 \mathrm{~cm}^{3}}$ or $\mathrm{D}_{0.1 \mathrm{~cm}^{3}}$. The rationale behind is that the point dose is clinically meaningless, and the dose to a volume may be better correlated with the side effects.

\section{Dose-volume limits for PTV and OARs}

In the BT community, no general agreement exists regarding the acceptable criteria for a good breast implant. In the GEC-ESTRO randomized study, the coverage index (CI) had to be larger than 0.9 , i.e. at least $90 \%$ of the PTV had to receive the PD [26]. There was a requirement for dose uniformity, namely the DNR $<0.35$. In the study, only skin was regarded as OAR, and the maximum surface dose had to be less than $70 \%$ of the PD. For reporting purpose, several additional parameters had to be recorded, but fulfilment of condition was not set up for them.
In the NSABP B-39/RTOG 0413 protocol for multi-catheter breast BT, the DHI for implant should be $\geq 0.75$, which corresponds to DNR $\leq 0.25$ [27]. In addition to relative values, an absolute high dose volumes are also limited. The volume of tissue receiving $150 \%$ and $200 \%$ of the PD should be less than or equal to $70 \mathrm{~cm}^{3}$ and $20 \mathrm{~cm}^{3}$, respectively. Regarding target coverage, the dose $\geq 90 \%$ of the PD must cover $\geq 90 \%$ of the PTV. For uninvolved normal breast, the limitation is that $<60 \%$ of the whole breast volume should receive $\geq 50 \%$ of the PD, and the skin surface dose should not exceed the PD. The dose to other organs including the heart, lung, and ribs is generally minimal, so no thresholds are used, however, recording few parameters for reporting is recommended. In a paper by Darby et al. [28], it was found that the risk of major cardiac events increased linearly by $7.4 \%$ per gray of mean heart dose, therefore, to characterize the dose to heart reporting the mean dose is recommended. In multicatheter breast BT, chest wall pain or rib fracture are rare events, but the doses should be kept as low as possible.

\section{Discussion}

Initially, CT imaging was used only for evaluation purpose after standard fluoroscopy-guided implantation $[12,13,14,15,29]$, but afterwards, the implantations themselves were performed using 3D CT information 
$[15,16,30,31,32,33]$. These and other studies confirmed that with introduction of image-guided implantation techniques, the treatment plans improved considerably compared to the traditional fluoroscopy-guided implantation and planning [11,34]. Cuttino et al. [15] reported that the percentage of patients satisfying with their dosimetric goals of target coverage and dose homogeneity increased from 42 to $93 \%$, when CT-guided technique was used instead of fluoroscopic guided free-handed catheter insertion technique. In their study, not only the coverage but also the DHI was better with the CT planning. Cholewka et al. [35] compared two different pre-planning methods (2D vs. 3D). In the first group of patients, the catheter positions were planned with X-ray fluoroscopy imaging (2D), while in the second group, the treatments were pre-planned with cone beam CT imaging (3D). For DVH-based plan evaluations, CT imaging was used for all patients. According to their results, the target coverage $\left(\mathrm{V}_{100}\right)$ was significantly higher for 3D pre-planning $(91.7 \%$ vs. $86.1 \%)$, and the dose distributions were more homogeneous (DHI: 0.60 vs. 0.53). The transition from classical X-ray film based planning to 3D planning was carried out step-by-step in many institutions, and the 3D dosimetry induced lots of challenges for radiation oncologists and medical physicists [18]. Furthermore, a learning curve can be observed during this process as it was well demonstrated by Cholewka et al. [36] and reported by Wiercinska et al. [18].

The quality of dose distributions in interstitial BT can be improved with the use of different dose optimization methods and image based 3D information for planning the geometry of the catheter positions $[12,13,14,37]$. Geometrically optimized implants are much more conformal than the non-optimized ones (PDS) with much less dose to normal tissues [19]. With conformal dose planning, the target volume coverage can be significantly increased compared to the traditional dosimetry systems, but the dose homogeneity worsens $[13,14,16,18]$. Geometrical optimization followed by graphical optimization can provide acceptable dose plans in most of the clinical cases $[18,32]$. In classical breast BT, the dosimetry was relied

A

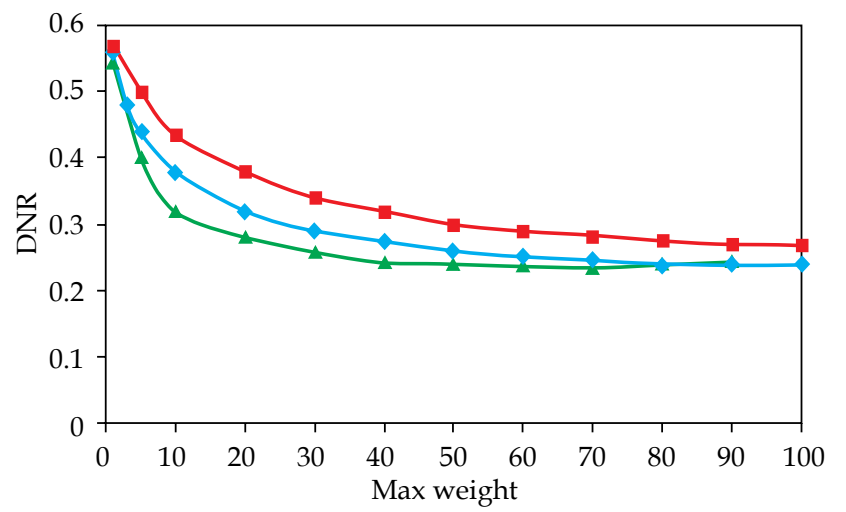

$\neg$ Min weight $=25 \quad \neg$ Min weight $=50$ on reference dose points, which were related to the catheters geometry. The dose points were placed either in the center points between catheters or at specified distances from the catheters in outer directions. In the former case, the prescription isodose was generally selected as $85 \%$ of the mean central dose like in the PDS, while with the latter technique, the dose was normalized to the mean dose in the dose points. In both cases, the target volume could only be approximated roughly. From this follows that the plan evaluation confined to the evaluation on dose uniformity in the implant volume and dose values in points.

Anatomy-based inverse optimization algorithms are already available in brachytherapy systems for clinical use $[22,23,24,38]$. During the optimization process, the source dwell times for active source dwell positions are calculated with the aim of fulfilling thresholds for dose-volume parameters. This is done by defining objectives and penalties for PTV and OARs. Then, an objective or cost function measuring how well the limits are fulfilled is minimized. Since the calculation is very time-consuming, in order to get the results in a reliable time, a powerful computer is needed for the clinical use.

The requirements for target coverage, dose homogeneity, and sparing of OARs are conflicting and to find the proper parameters and weight factors is a challenge for the physicist. This is demonstrated in Figure 4, where dose homogeneity characterized by the DNR and target coverage denoted by $\mathrm{V}_{100}$ are shown in the function of weight factor of maximum dose value (Max Weight). The calculations were performed in the Oncentra Brachy version 4.3 planning system, and the OARs were not taken into account. We note, that the weight factor for maximum dose (Max Weight and Max Value in Figure 3) controls the homogeneity, and the weight factor for minimum dose (Min Weight and Min Value in Figure 3) influences coverage. Figure $4 \mathrm{~A}$ shows that by increasing the weight factor for maximum dose, the DNR is becoming less meaning better homogeneity. However, at the same time, the $V_{100}$ is also decreasing and the target coverage is worsening. To select the proper weight factor, it requires clinical judgement, balancing between dose homogeneity

B

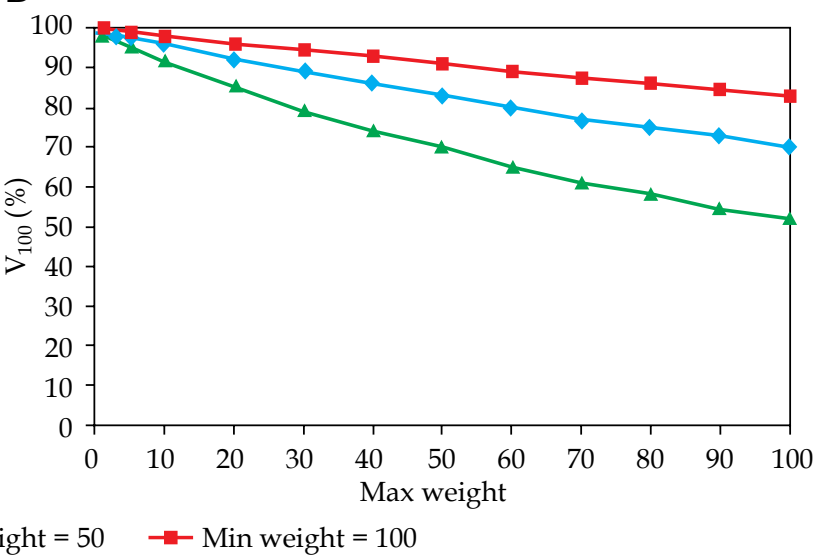

Fig. 4. Dependence of dose homogeneity (A) and target coverage (B) on the weight factor for maximum dose (Max Weight) in the planning target volume (PTV). DNR is the dose non-uniformity ratio. Min Weight is a weight factor, which controls the target coverage through minimum dose at the periphery of the PTV 
and target coverage. A weight factor in the range between 20-40 seems to be an appropriate choice with low DNR and concurrently high $\mathrm{V}_{100}$. From the Figure 4, the role of minimum dose factor (Min Weight) can be observed. By using a higher minimum dose factor, the coverage is better (red line in Figure 4B), but simultaneously, the dose will be less homogeneous (red line in Figure 4A). At first sight, the inverse planning seems to be an easy task, but it requires some experience to find those input parameters, which will result in clinically acceptable dose distribution. In Figure 5A, qualitative comparison between dose distributions of forward and inverse planning is presented in a CT slice. Regarding target coverage, the difference is negligible, but the high dose volume surrounded by the $150 \%$ is less with the HIPO (Figure 5B) resulting in better dose homogeneity. Table 3 shows a quantitative comparison between dosimetric parameters calculated for fifteen treatment plans made with geometrical optimization, followed by graphical optimization (GEOM+GRAPH) and HIPO. At the same target coverage $(91 \%)$, the HIPO plans were superior over the forward plans regarding all other parameters. The better conformality (COIN: 0.77 vs. 0.71 ) resulted in $29 \%$ less high dose volume $\left(\mathrm{V}_{100}\right)$ in the ipsilateral non-target breast. The dose homogeneity improved (DNR: 0.35 vs. 0.37 ), and dose to OARs was less by percentage values in the range of $1-15 \%$. Based on our initial experience and quantitative data presented here, we can claim that HIPO is an optimization tool that can produce clinically sound treatment plans at a significantly reduced overall planning and optimization time.

Nowadays, quantitative dose-volume histogram based evaluation of treatment plans is proposed. However, there is no consensus on which parameters should be reported. For the PTV, the minimum requirement is to report the $\mathrm{CI}$ or $\mathrm{V}_{100}$, DHI or DNR, and the COIN.

In our department in line with the GEC-ESTRO study, we intend to have at least $90 \%$ PTV coverage by the prescribed dose $\left(\mathrm{V}_{100}\right)$, while in the NSABP B-39/RTOG 0413 protocol, the $90 \%$ of the prescribed dose should cover at
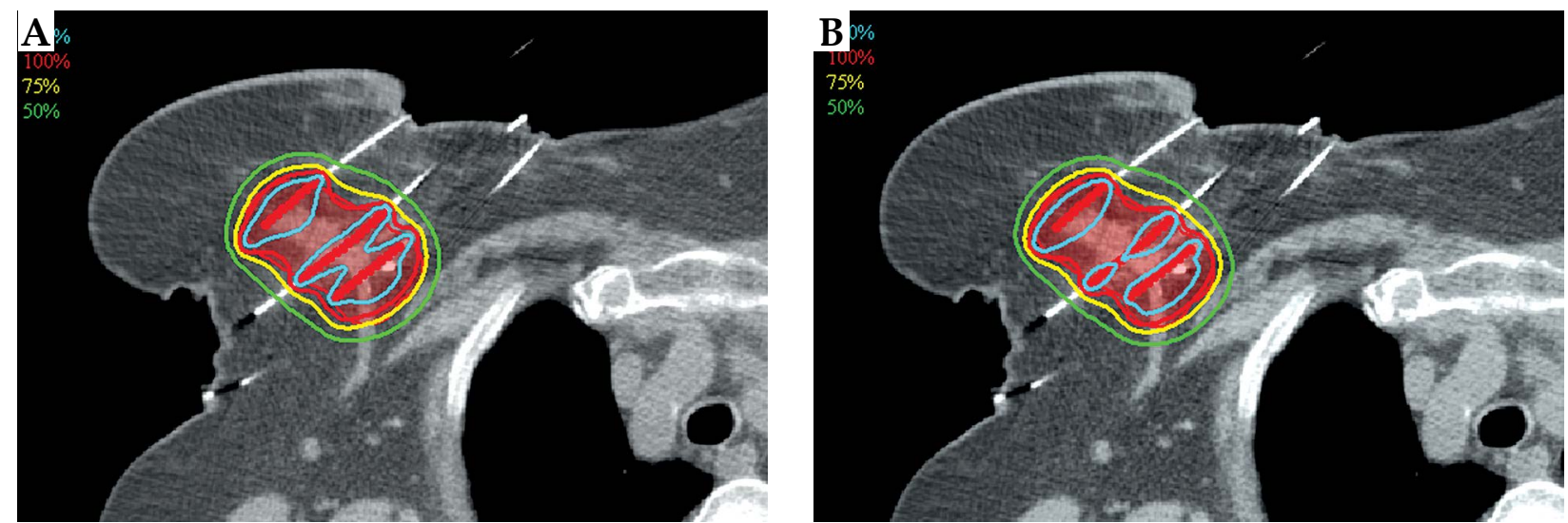

Fig. 5. Representative relative dose distributions on an axial computed tomography (CT) slice in treatment plan made with A) forward planning (GEOM+GRAPH), and with B) inverse planning (HIPO). The planning target volume (PTV) is shown in colour wash. More homogeneous dose distribution can be observed in the HIPO plan

Table 3. Dosimetrical comparison of treatment plans made by forward planning using geometrical optimization followed by graphical optimization (GEOM+GRAPH) and inverse optimization (HIPO). The percentage difference is related to GEOM+GRAPH plans

\begin{tabular}{lccc} 
& $\begin{array}{c}\text { Forward planning } \\
\text { GEOM + GRAPH }\end{array}$ & $\begin{array}{c}\text { Inverse planning } \\
\text { HIPO }\end{array}$ & Difference (\%) \\
\hline PTV $-\mathrm{V}_{100}(\%)$ & 91 & 91 & 0 \\
\hline Non-target breast $-\mathrm{V}_{100}(\%)$ & 1.7 & 1.2 & -29 \\
\hline COIN & 0.71 & 0.77 & +8 \\
\hline DNR & 0.37 & 0.35 & -6 \\
\hline Ipsilateral lung $-\mathrm{D}_{1 \mathrm{~cm}^{3}}(\%)$ & 35.8 & 34.0 & -5 \\
\hline Skin $-\mathrm{D}_{1 \mathrm{~cm}}(\%)$ & 52.5 & 52.0 & -1 \\
\hline Ribs $-\mathrm{D}_{1 \mathrm{~cm}}(\%)$ & 41.4 & 37.5 & -9 \\
\hline Heart $-\mathrm{D}_{1 \mathrm{~cm}}{ }^{3}(\%)$ & 18.0 & 15.3 & -15 \\
\hline Contralateral lung $-\mathrm{D}_{1 \mathrm{~cm}^{3}}(\%)$ & 3.7 & 3.4 & -8 \\
\hline Contralateral breast $-\mathrm{D}_{1 \mathrm{~cm}^{3}}(\%)$ & 3.0 & 2.6 & -13
\end{tabular}


A

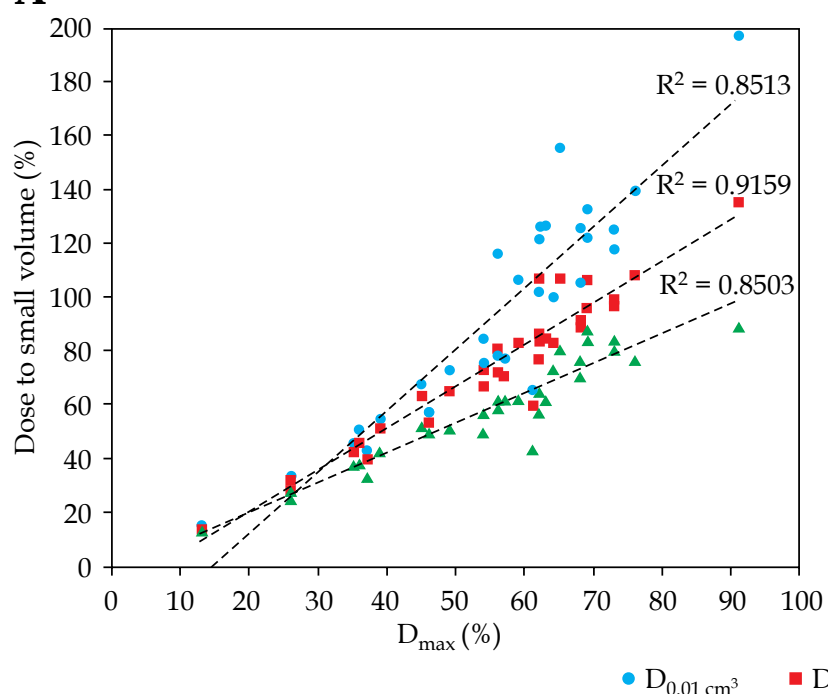

B

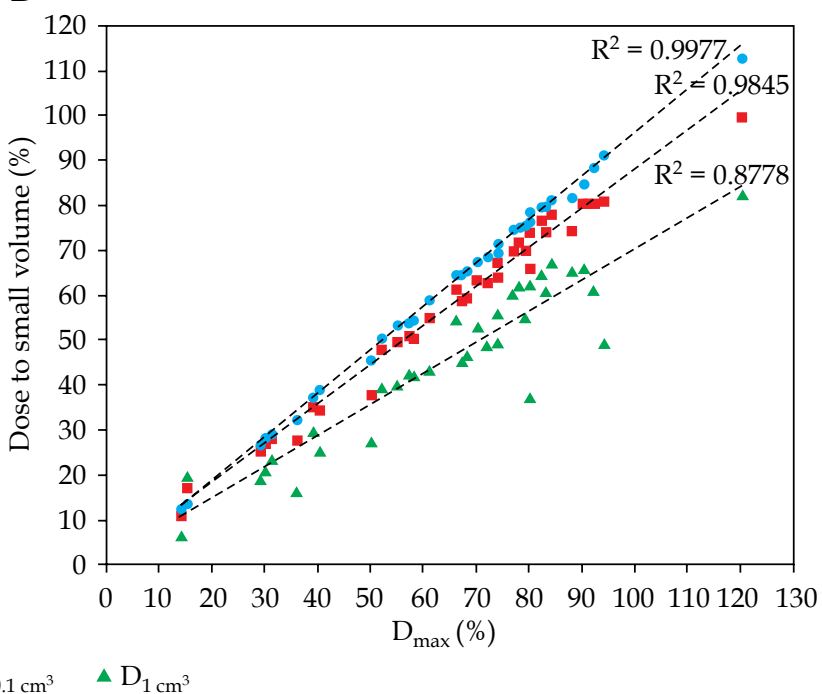

Fig. 6. Correlation between the dose to the most exposed small volumes and maximum point dose for the A) skin, and $\mathbf{B}$ ) ribs

least $90 \%$ of the PTV. With the latter constraint, a lower DNR value can be obtained (DNR < 0.25). Conformality is rarely investigated in breast BT, and there is no consensus on what degree of conformality is acceptable or desirable. Pieters et al. [37] obtained a conformality number of 0.48 at a typical breast implant with geometrical optimization. Kolotas et al. [30] reported COIN value of 0.76 for 42 breast implants, while Baltas et al. [25] aimed to achieve a COIN value above 0.64 by using their CTbased planning system. In a study by Major et al. [32], the mean COIN was 0.68 (range, 0.51-0.82) calculated for 49 patients. Similar COINs were reported by Cholewka et al. [36] and Gurran et al. [39] with the values of 0.69 (range, $0.37-0.84$ ) and 0.67 (range, 0.59-0.87), respectively.

Reporting the dose to OARs is particularly not uniform at all. From this follows that the dose-volume limits can be very different for the various parameters. Moreover, no common guidelines are available on how to define the skin dose or the skin as an organ. Berger et al. [40] investigated DVH parameters for skin dose, and they recommended a more sophisticated way to report organ dose than using point doses only. Similarly, Lasota et al. [41] used different skin models to estimate the dose to skin, and they concluded that large differences exist between models using various skin contouring protocols. Hilts et al. [42] recommend using $\mathrm{D}_{0.2 \mathrm{~cm}^{3}}$ for a $2-\mathrm{mm}$ thick skin layer as a surrogate for dose to skin area of $1 \mathrm{~cm}^{2}$. In a paper of Lettmaier et al. [43], dose to $1 \mathrm{~cm}^{3}$ and $0.1 \mathrm{~cm}^{3}$ of skin volume with $5 \mathrm{~mm}$ thickness were reported. Gifford et al. [44] has found that large differences in skin volume definition leads to confusion and can result in significant dose differences, making the dosimetric comparisons and correlations with toxicities difficult or impossible. Traditionally, the dose to skin was characterized with the maximum surface dose, which is a point dose. In a recent paper by Major et al. [45], doses to the most exposed small volumes in OARs are reported. To make the intercomparisons between point doses and volumetric doses, a correlation analysis should be made. Figure 6 presents the correlation between the dose to small volumes and maximum point dose for skin and ribs calculated for thirty-four breast implant patients. The skin was outlined as a 5-mm shell inside the body, and the maximum skin dose was calculated on the outer surface, while the maximum dose in ribs was calculated inside the organ. Regarding the skin, the $D_{0.01 \mathrm{~cm}^{3}}$ is not a good substitute for $\mathrm{D}_{\max }$ $\left(R^{2}=0.8513\right)$, because the $D_{\max }$ is always on the outer surface of the skin volume, while the high dose volumes (e.g. $0.01 \mathrm{~cm}^{3}$ ) develop in the inner part of the skin closer to the source dwell positions and the corresponding doses can be different. Better correlation was found for the $\mathrm{D}_{0.1 \mathrm{~cm}^{3}}$ $\left(R^{2}=0.9159\right)$. For the ribs, an excellent correlation $\left(R^{2}=\right.$ 0.9977 ) can be observed between $D_{0.01 \mathrm{~cm}^{3}}$ and $D_{\text {max }}$, since these two values are nearly always identical. The $D_{\max }$ is always inside the most exposed volume of $0.01 \mathrm{~cm}^{3}$. For larger volumes $\left(0.1 \mathrm{~cm}^{3}, 1 \mathrm{~cm}^{3}\right)$, the correlation is weaker, but still high.

\section{Future directions}

Similarly to EBRT, inverse planning is expected to be used in BT in daily clinical practice very soon, especially in multicatheter breast implants. Its advantages are obvious with the improved dosimetry and quicker calculation time. However, the protocols with the dose-volume constraints used during the optimization still should be defined. For a plan evaluation, the use of volumetric dose parameters is already recommended, and will be obligatory in the future in order to find a correlation between dosimetry and clinical outcome. However, a consensus is needed on which parameters should be reported, and which can have real clinical significance.

\section{Disclosure}

Authors report no conflict of interest.

\section{References}

1. Fijuth J. Brachytherapy in breast cancer. J Contemp Brachytherapy 2009; 1: 117-120. 
2. Skowronek J, Wawrzyniak-Hojczyk M, Ambrochowicz K Brachytherapy in accelerated partial breast irradiation (APBI) - review of treatment methods. J Contemp Brachytherapy 2012; 4: 152-164.

3. Offersen BV, Overgaard M, Kroman N et al. Accelerated partial breast irradiation as part of breast conserving therapy of early breast carcinoma: A systematic review. Radiother Oncol 2009; 90: 1-13.

4. Njeh CF, Saunders MW, Langton CM. Accelerated partial breast irradiation using external beam conformal radiation therapy: A review. Crit Rev Oncol Hematol 2012; 81: 1-20.

5. Strnad V, Ott OJ, Hildebrandt G et al. 5-year results of accelerated partial breast irradiation using sole interstitial multicatheter brachytherapy versus whole-breast irradiation with boost after breast-conserving surgery for low-risk invasive and in-situ carcinoma of the female breast: a randomized, phase 3, non-inferiority trial. Lancet 2016; 387: 229-238.

6. Polgár C, van Limbergen E, Pötter R et al. Patient selection for accelerated partial breast irradiation (APBI) after breastconserving surgery: Recommendations of the Groupe Européen de Curiethérapie-European Society for Therapeutic Radiology and Oncology (GEC-ESTRO) Breast Cancer Working Group based on clinical evidence (2009). Radiother Oncol 2010; 94: 264-273.

7. Smith BD, Arthur DW, Buchholz TA et al. Accelerated partial breast irradiation consensus statement from the American Society for Radiation Oncology (ASTRO). Int J Radiat Oncol Biol Phys 2009; 74: 987-1001.

8. Shah C, Wobb J, Manyam B et al. Accelerated partial breast irradiation utilizing brachytherapy: patient selection and workflow. J Contemp Brachytherapy 2016; 8: 90-94.

9. Strnad V, Hannoun-Levi JM, Guinot JL et al. Recommendations from GEC ESTRO Breast Cancer Working Group (I): Target definition and target delineation for accelerated or boost Partial Breast Irradiation using multicatheter interstitial brachytherapy after breast conserving closed cavity surgery. Radiother Oncol 2015; 115: 342-348.

10. Major T, Gutiérrez C, Guix B et al. On behalf of Breast Cancer Working Group of GEC-ESTRO. Recommendations from GEC ESTRO Breast Cancer Working Group (II): Target definition and target delineation for accelerated or boost Partial Breast Irradiation using multicatheter interstitial brachytherapy after breast conserving open cavity surgery. Radiother Oncol 2016; 18: 199-204.

11. Vicini FA, Jaffray DA, Horwitz EM et al. Implementation of 3D-virtual brachytherapy in the management of breast cancer: a description of a new method of interstitial brachytherapy. Int J Radiat Oncol Biol Phys 1998; 40: 629-635.

12. Vicini FA, Kestin LL, Edmundson GK et al. Dose-volume analysis for quality assurance of interstitial brachytherapy for breast cancer. Int J Radiat Oncol Biol Phys 1999; 45: 803-810.

13. Kestin LL, Jaffray DA, Edmundson GK et al. Improving the dosimetric coverage of interstitial high-dose-rate breast implants. Int J Radiat Oncol Biol Phys 2000; 46: 35-43.

14. Major T, Fodor J, Takácsi-Nagy Z et al. Evaluation of HDR interstitial breast implants planned by conventional and optimized CT-based dosimetry systems with respect to dose homogeneity and conformality. Strahlenther Onkol 2005; 181: 89-96.

15. Cuttino LW, Todor D, Arthur DW. CT-guided multi-catheter insertion technique for partial breast brachytherapy: Reliable target coverage and dose homogeneity. Brachytherapy 2005; 4: 10-17.

16. Major T, Fröhlich G, Lövey K et al. Dosimetric experience with accelerated partial breast irradiation using image-guided interstitial brachytherapy. Radiother Oncol 2009; 90: 48-55.

17. Gutiérrez C, Najjari D, Martínez E et al. The use of an interstitial boost in the conservative treatment of breast cancer: how to perform it routinely in a radiotherapy department. J Contemp Brachytherapy 2014; 6: 397-403.

18. Wiercinska J, Wronczewska A, Kabacinska R et al. Transition from Paris dosimetry system to 3D image-guided planning in interstitial breast brachytherapy. J Contemp Brachytherapy 2015; 7: 479-484.

19. Major T, Polgár C, Fodor J et al. Evaluation of geometrically optimized single- and double-plane interstitial high dose rate implants with respect to conformality and homogeneity. Acta Oncol 2003; 42: 15-21.

20. Van der Laarse R. The stepping source dosimetry system as an extension of the Paris system. In: Brachytherapy from radium to optimization. Mould RF, Battermann JJ, Martinez AA, Speiser BL (eds.). Nucletron BV, Veenendaal 1994; pp. 319-330.

21. Van der Laarse R, Prins TPE. Comparing the stepping source dosimetry system and the Paris system using volume-dose histograms of breast implants. In: Brachytherapy from radium to optimization. Mould RF, Battermann JJ, Martinez AA, Speiser BL (eds.). Nucletron BV, Veenendaal 1994; pp. 352-372.

22. Karabis A, Giannouli S, Baltas D. HIPO: A hybrid inverse treatment planning optimization algorithm in HDR brachytherapy. Radiother Oncol 2005; 76: S29.

23. Jamema SV, Sharma S, Mahantshetty U et al. Comparison of IPSA with dose-point optimization and manual optimization for interstitial template brachytherapy for gynecologic cancers. Brachytherapy 2010; 10: 306-312.

24. Dinkla AM, van der Laarse R, Kaljouw E et al. A comparison of inverse optimization algorithms for HDR/PDR prostate brachytherapy treatment planning. Brachytherapy 2015; 14:279-288.

25. Baltas D, Kolotas C, Geramani K et al. A conformal index (COIN) to evaluate implant quality and dose specification in brachytherapy. Int J Radiat Oncol Biol Phys 1998; 40: 515-524.

26. Strnad V, Polgár C. Phase III Multicenter Trial: Interstitial brachytherapy alone versus external beam radiation therapy after breast conserving surgery for low risk invasive carcinoma and low risk duct carcinoma in situ (DCIS) of the female breast. Study Protocol, European Brachytherapy Breast Cancer GEC-ESTRO Working Group, 2004; available at: http:/ / www.apbi.uni-erlangen.de/outline/outline.html, Accessed 16 December, 2016.

27. Vicini F, White J, Arthur D et al. NSABP Protocol B39/ RTOG Protocol 0413: A randomized phase III study of conventional whole breast irradiation versus partial breast irradiation for women with stage 0.I, or II breast cancer. National Surgical Adjuvant Breast and Bowel Project/ Radiation Therapy Oncology Group, Pittsburgh/Philadelphia, 2004; available at: http:/ / atc.wustl.edu/protocols/nsabp/b-39/0413.pdf, Accessed 16 December, 2016.

28. Darby SC, Ewertz M, McGale P et al. Risk of ischemic heart disease in women after radiotherapy for breast cancer. $N$ Engl J Med 2013; 368: 987-998.

29. Weed DW, Edmundson K, Vicini FA et al. Accelerated partial breast irradiation: a dosimetric comparison of three different techniques. Brachytherapy 2005; 4: 121-129.

30. Kolotas C, Baltas D, Zamboglou N. CT-based interstitial HDR brachytherapy. Strahlenther Onkol 1999; 175: 419-427.

31. Das RK, Patel R, Shah $\mathrm{H}$ et al. 3D CT-based high-doserate breast brachytherapy implants: Treatment planning and quality assurance. Int J Radiat Oncol Biol Phys 2004; 59: 1224-1228.

32. Major T, Polgár Cs, Lövey K et al. Dosimetric characteristics of accelerated partial breast irradiation with CT image-based multicatheter interstitial brachytherapy: A single institution's experience. Brachytherapy 2011; 10: 421-426. 
33. Tang JI, Tan PW, Koh VY et al. Multi-catheter interstitial accelerated partial breast irradiation - tips and tricks for a good insertion. J Contemp Brachytherapy 2014; 6: 85-90.

34. Polgár C, Major T, Somogyi A et al. CT-image based conformal brachytherapy of breast cancer: the significance of semi3-D and 3-D treatment planning. Strahlenther Onkol 2000; 176: 118-124.

35. Cholewka A, Szlag M, Slosarek K et al. Comparison of 2Dand 3D-guided implantation in accelerated partial breast irradiation (APBI). J Contemp Brachytherapy 2009; 1: 207-210.

36. Cholewka A, Szlag M, Białas B et al. The importance of the implant quality in APBI - Gliwice experience. Dosimetric evaluation. J Contemp Brachytherapy 2013; 54: 227-231.

37. Pieters BR, Saarnak AE, Steggerda MJ et al. A method to improve the dose distribution of interstitial breast implants using geometrically optimized stepping source techniques and dose normalization. Radiother Oncol 2001; 58: 63-70.

38. Lessard E, Pouliot J. Inverse planning anatomy-based dose optimization for HDR-brachytherapy of the prostate using fast simulated annealing algorithm and dedicated objective function. Med Phys 2001; 28: 773-779.

39. Gurran L, Wadasadawala T, Joshi K et al. Multi-catheter interstitial brachytherapy for partial breast irradiation: an audit of implant quality based on dosimetric evaluation comparing intra-operative versus post-operative placement. J Contemp Brachytherapy 2016; 8: 116-121.

40. Berger D, Kauer-Dorner D, Seitz W et al. Concepts for critical organ dosimetry in three-dimensional image-based breast brachytherapy. Brachytherapy 2008; 7: 320-326.

41. Lasota J, Kabacinska R, Makarewicz R. Dose estimation for different skin models in interstitial breast brachytherapy. J Contemp Brachytherapy 2014; 6: 200-207.

42. Hilts M, Halperin H, Morton D et al. Skin dose in breast brachytherapy: Defining a robust metric. Brachytherapy 2015; 14: $970-978$.

43. Lettmaier S, Kreppner S, Lotter M et al. Radiation exposure of the heart, lung, and skin by radiation therapy for breast cancer: A dosimetric comparison between partial breast irradiation using multicatheter brachytherapy and whole breast teletherapy. Radiother Oncol 2011; 100: 189-194.

44. Gifford KA, Pacha O, Hebert AA et al. A new paradigm for calculating skin dose. Brachytherapy 2013; 12: 114-119.

45. Major T, Stelczer G, Pesznyák C et al. Multicatheter interstitial brachytherapy versus intensity modulated external beam therapy for accelerated partial breast irradiation: A comparative treatment planning study with respect to dosimetry of organs at risk. Radiother Oncol 2017; 122: 17-23. 